\title{
Estimation of Heterosis for Green Pod Yield and Attributing Characters in Cowpea (Vigna unguiculata (L.) Walp.)
}

\author{
Jyoti Kumari* and D.A. Chauhan \\ Department of Genetics and Plant Breeding, Navsari Agricultural University, \\ Navsari, Gujarat- 396 450, India \\ *Corresponding author
}

Key w o r d s
Cowpea,
Heteobeltiosis,
Standard heterosis,
Green pod yield per
plant

\section{Introduction}

Cowpea [Vigna unguiculata (L.) Walp.] is diploid crop with chromosome number of $(2 \mathrm{n}=22)$, belongs to family Fabaceae, one of the oldest source of human food, in form of green pods as well as grains has most likely been used as a crop. It is native of West Africa (Vavilov, 1951) but Steele (1976) suggested Ethiopia as the primary and Africa as the secondary centre of diversity. Among all the pulses, cowpea locally known as lobiya, chowla (chowli), southern pea or black eye pea, is an annual legume that is adopted to warm condition and cultivated in the tropics and sub-tropics for dry grains, green edible pods for vegetable as well as fodder. Development of cultivars with early maturity, acceptable grain as well as vegetable quality, resistance to some important diseases and pests have significantly increased the yield and cultivated area. The overall effect of plant breeding on genetic diversity has been a long standing concern in the evolutionary biology 
of crop plants (Simmonds, 1962). Selfpollinating crop like cowpea, variability is often created through hybridization between carefully chosen parents. The scope of exploitation of hybrid vigour will depend on the direction and magnitude of heterosis, biological feasibilities and the type of gene action involved. The information of such estimates is essential to plan efficient breeding programme for the improvement of crop. Although, the hybrid vigour cannot be exploited commercially in highly selfpollinated crop like cowpea, the heterotic $\mathrm{F}_{1}$ 's can be used to isolate a higher frequency of productive derivatives in their later generations.

\section{Materials and Methods}

Thirty hybrids were developed by adopting full diallel mating design. The experimental material for the present investigation consisted of 38 entries including six parents(NCK-15-9, NCK-15-10, NC-15-41, NC-15-42, NC-15-44, and NC-15-45) and resultant 30 crosses (full diallel) along with GC-3 and GDVC-2 as checks. The seeds of these entries were obtained from Pulse Research Station, Navsari Agricultural University, Navsari. These parents were selected carefully on the basis of earlier reports as well as the observations recorded at the centre. For obtaining hybrid seeds, these six parents were sown at Main Pulse Research Station, NAU, Navsari during summer 2017. All possible single crosses (including reciprocals) were made to complete the $6 \times 6$ full diallel set. Hand emasculation and pollination methods were adopted. All the hybrids and self-seeds of parents were stored properly in seed packets for sowing in the kharif season 2017-18. The observations were recorded on parents and $F_{1}$ 's for fifteen quantitative traits and one quality traits viz. Days to 50 per cent flowering, plant height $(\mathrm{cm})$, primary branches per plant, pods per plant, pod length $(\mathrm{cm})$, seeds per pod, green seed weight $(\mathrm{g})$, green pod yield per plant $(\mathrm{g})$, shelling percentage (per cent) and protein content (per cent). Heterosis expressed as per cent increase or decrease in the mean value of $\mathrm{F}_{1}$ hybrid over mid parent (heterosis), better parent (heterobeltiosis) and over standard check (standard heterosis) was calculated for various characters over environments following procedure given by Fonseca and Patterson (1968).

\section{Results and Discussion}

Heterosis is of direct relevance for developing hybrids in cross-pollinated as well as in selfpollinated crops. But, exploitation of heterosis in cowpea has limited application because of practical difficulties for emasculation, pollination of flowers, difficulties of seed set and hybrid seed production in sufficient quantity. However, magnitude of heterosis provides information on the extent of genetic diversity of parents involved in a cross and helps to choose the parents in developing superior $F_{1}$ 's so as to exploit the hybrid vigour. The nature and magnitude of heterosis can help in identifying superior cross combinations and their exploitation to get better transgressive segregants (Arunachalam, 1976). The knowledge of heterosis would also help in elimination of poor crosses in early generation of testing itself. Heterosis is a function of number of loci at which the parents carry different alleles and the magnitude and direction of the non-additive effects within or between those loci in hybrid combinations (Jinks, 1953).

Hetrobeltiosis and standard heterosis of some important characters over all the three location and pooled basis is depicted in table 1 to 4 . The spectrum of variation in heterobeltiosis and standard heterosis for all the characters and the number of crosses depicting heterosis in desirable directions is presented in table 5 and top ten crosses as per higher heterosis and highest per se performance are depicted in table 6 . 
Table.1 Extent of heterobeltiosis and standard heterosis in per cent for days to 50 per cent flowering in different environments and pooled over environments in cowpea

\begin{tabular}{|c|c|c|c|c|c|c|c|c|c|c|c|c|c|}
\hline \multirow{2}{*}{$\begin{array}{l}\text { Sr. } \\
\text { no. }\end{array}$} & \multirow[t]{2}{*}{ Crosses } & \multicolumn{3}{|c|}{ Navsari } & \multicolumn{3}{|c|}{ Mangrol } & \multicolumn{3}{|c|}{ Achhalia } & \multicolumn{3}{|c|}{ Pooled } \\
\hline & & BP & SC1 & SC2 & $\mathbf{B P}$ & SC1 & SC2 & $\mathbf{B P}$ & SC1 & SC2 & $\mathbf{B P}$ & SC1 & SC2 \\
\hline \multicolumn{14}{|c|}{ Direct Crosses } \\
\hline 1 & NCK-15-9 x NCK-15-10 & -2.36 & $11.11 * *$ & $7.73 *$ & -2.33 & 7.15 & 0.01 & -3.33 & 6.56 & 0.15 & -2.69 & $8.22 * *$ & 2.48 \\
\hline 2 & NCK-15-9 x NC-15-41 & $-10.63 *$ & $9.68 *$ & 6.35 & -7.48 & 6.39 & -0.71 & -0.5 & -6.56 & $-12.19 * *$ & $-6.16^{*}$ & 2.89 & -2.56 \\
\hline 3 & NCK-15-9 x NC-15-42 & $9.06 *$ & -1.28 & -4.28 & $13.71 * *$ & -4.71 & $-11.07 * *$ & $9.06 *$ & -1.72 & -7.64 & $12.67 * *$ & -2.56 & $-7.72 * *$ \\
\hline 4 & NCK-15-9 x NC-15-44 & $10.98 * *$ & -2.99 & -5.94 & $11.61 * *$ & -2.4 & $-8.91 *$ & 7.11 & 0.39 & -5.65 & $11.82 * *$ & -1.61 & $-6.82 * *$ \\
\hline 5 & NCK-15-9 x NC-15-45 & $-8.77^{*}$ & 5.98 & 2.76 & $-14.17 * *$ & 5.72 & -1.33 & 0.79 & -2.34 & $-8.22 *$ & $-7.03^{* *}$ & 2.97 & -2.49 \\
\hline 6 & NCK-15-10 x NC-15-41 & -2.16 & 1.28 & -1.8 & 2.63 & -3.62 & $-10.05^{* *}$ & -5.88 & -1.56 & -7.49 & -1.81 & -1.32 & $-6.55 * *$ \\
\hline 7 & NCK-15-10 x NC-15-42 & 7.09 & 0.85 & -2.21 & 6.51 & -2.1 & $-8.63 *$ & -3.03 & 6.25 & -0.15 & 3.42 & 1.79 & -3.61 \\
\hline 8 & NCK-15-10 x NC-15-44 & 4.53 & 3.63 & 0.48 & 0.28 & 4.42 & -2.55 & -0.38 & 3.52 & -2.72 & 1.46 & 3.85 & -1.66 \\
\hline 9 & NCK-15-10 x NC-15-45 & -3.77 & 1.11 & -1.96 & $-15.17 * *$ & 6.64 & -0.47 & $-13.49 * *$ & $11.72 * *$ & 4.99 & $-10.85 * *$ & $6.6^{* *}$ & 1.00 \\
\hline 10 & NC-15-41 x NC-15-42 & -7.33 & 6.41 & 3.17 & -1.36 & 0.34 & -6.36 & -3.28 & -3.98 & $-9.77 *$ & -3.97 & 0.77 & -4.57 \\
\hline 11 & NC-15-41 x NC-15-44 & 6.25 & -7.05 & $-9.88^{*}$ & -0.3 & -0.71 & -7.34 & -2.52 & -4.69 & $-10.43^{*}$ & 1.11 & -4.15 & $-9.23 * *$ \\
\hline 12 & NC-15-41 x NC-15-45 & -3.07 & 0.43 & -2.63 & $-10.72 * *$ & 2.52 & -4.32 & -0.25 & -6.8 & $-12.41 * *$ & -2.46 & -1.43 & $-6.65 * *$ \\
\hline 13 & NC-15-42 x NC-15-44 & -5.71 & $14.74 * *$ & $11.25 * *$ & 0.55 & $13.58 * *$ & 6.00 & 5.04 & 4.61 & -1.69 & 0.76 & $10.80^{* *}$ & 4.93 \\
\hline 14 & NC-15-42 x NC-15-45 & $-16.23 * *$ & $13.25 * *$ & $9.80 *$ & -3.95 & -3.74 & $-10.16^{* *}$ & 0.4 & -1.95 & -7.86 & $-6.38 *$ & 2.35 & -3.08 \\
\hline 15 & NC-15-44 x NC-15-45 & 2.41 & -4.91 & $-7.81 *$ & -3.22 & -4.42 & $-10.79 * *$ & 5.16 & -6.64 & $-12.26^{* *}$ & 1.63 & $-5.36^{*}$ & $-10.38 * *$ \\
\hline
\end{tabular}


Table 1 Contd......

\begin{tabular}{|c|c|c|c|c|c|c|c|c|c|c|c|c|c|}
\hline \multirow{2}{*}{$\begin{array}{l}\text { Sr. } \\
\text { no. }\end{array}$} & \multirow[t]{2}{*}{ Crosses } & \multicolumn{3}{|c|}{ Navsari } & \multicolumn{3}{|c|}{ Mangrol } & \multicolumn{3}{|c|}{ Achhalia } & \multicolumn{3}{|c|}{ Pooled } \\
\hline & & BP & SC1 & SC2 & BP & SC1 & SC2 & BP & SC1 & SC2 & BP & SC1 & SC2 \\
\hline \multicolumn{14}{|c|}{ Reciprocal Crosses } \\
\hline 16 & NCK-15-10 x NCK-15-9 & 1.18 & 7.26 & 4 & 0.08 & 4.63 & -2.35 & 2.12 & 0.94 & -5.14 & 1.15 & 4.18 & -1.35 \\
\hline 17 & NC-15-41 x NCK-15-9 & $-8.62 *$ & 7.69 & 4.42 & -4.08 & 3.03 & -3.85 & -5.55 & -1.87 & -7.78 & $-6.07 *$ & 2.8 & -2.65 \\
\hline 18 & NC-15-41 x NCK-15-10 & 5.6 & -6.41 & $-9.26 *$ & 5.18 & -6.14 & $-12.40 * *$ & 3.11 & $-9.92 *$ & $-15.35^{* *}$ & 4.62 & $-7.56^{* *}$ & $-12.46 * *$ \\
\hline 19 & NC-15-42 x NCK-15-9 & $12.20 * *$ & -4.7 & -7.6 & $7.62 *$ & 2.02 & -4.79 & $10.50 * *$ & -3.28 & $-9.10 *$ & $12.17 * *$ & -2.01 & $-7.20 * *$ \\
\hline 20 & NC-15-42 x NCK-15-10 & $11.81 * *$ & -4.27 & -7.18 & $11.33 * *$ & -7.15 & $-13.34 * *$ & $8.86^{*}$ & -6.02 & $-11.67 * *$ & $10.64 * *$ & $-5.83 *$ & $-10.82 * *$ \\
\hline 21 & NC-15-42 x NC-15-41 & -6.29 & 5.38 & 2.18 & -4.08 & 3.03 & -3.85 & $-19.75 * *$ & $11.33^{*}$ & 4.63 & $-10.09 * *$ & $6.71 * *$ & 1.05 \\
\hline 22 & NC-15-44 x NCK-15-9 & 17.84 & $-10.47 *$ & $-13.19 * *$ & $18.47 * *$ & $-9.97 *$ & $-15.97 * *$ & $13.61 * *$ & -6.64 & $-12.26 * *$ & $18.41 * *$ & $-8.96^{* *}$ & $-13.78 * *$ \\
\hline 23 & NC-15-44 x NCK-15-10 & 6.89 & 1.07 & -2.01 & 3.49 & 1.05 & -5.69 & $8.71 *$ & -5.86 & $-11.53^{* *}$ & $6.41 * *$ & -1.37 & $-6.60 * *$ \\
\hline 24 & NC-15-44 x NC-15-41 & -0.65 & -0.21 & -3.25 & -3.44 & 2.4 & -4.43 & -0.42 & -6.64 & $-12.26 * *$ & -1.5 & -1.62 & $-6.84 * *$ \\
\hline 25 & NC-15-44 x NC-15-42 & 3.74 & 4.49 & 1.31 & $9.39 *$ & 3.49 & -3.41 & $13.76 * *$ & -5 & $-10.72 *$ & $9.70 * *$ & 0.82 & -4.52 \\
\hline 26 & NC-15-45 x NCK-15-9 & 1.32 & -3.85 & -6.77 & 4 & $-11.10^{* *}$ & $-17.03 * *$ & 8.1 & $-9.53 *$ & $-14.98 * *$ & 4.6 & $-8.22 * *$ & $-13.08 * *$ \\
\hline 27 & NC-15-45 x NCK-15-10 & 1.75 & -4.27 & -7.18 & $-18.19 * *$ & $9.44 *$ & 2.14 & -3.44 & 1.82 & -4.31 & $-6.39 *$ & 2.35 & -3.07 \\
\hline 28 & NC-15-45 x NC-15-41 & $-8.42 *$ & 5.64 & 2.43 & -4.9 & -2.86 & $-9.34 *$ & $14.87 * *$ & $-20.86^{* *}$ & $-25.62 * *$ & 2.77 & $-6.46 * *$ & $-11.42 * *$ \\
\hline 29 & NC-15-45 x NC-15-42 & $-13.60 * *$ & $10.68 * *$ & 7.32 & $-9.13 *$ & 1.05 & -5.69 & $16.67 * *$ & $-17.97 * *$ & $-22.91 * *$ & -1.3 & -2.54 & $-7.71 * *$ \\
\hline \multirow[t]{4}{*}{30} & NC-15-45 x NC-15-44 & -1.97 & -0.64 & -3.66 & $-9.49 *$ & 1.39 & -5.38 & $13.10 * *$ & $-14.45 * *$ & $-19.60 * *$ & 1.09 & $-4.84 *$ & $-9.88 * *$ \\
\hline & S.E.(d) \pm & 1.56 & 1.56 & 1.56 & 1.61 & 1.61 & 1.61 & 1.87 & 1.87 & 1.87 & 1.87 & 0.96 & 0.96 \\
\hline & CD @ 0.05 & 3.1 & 3.1 & 3.1 & 3.21 & 3.21 & 3.21 & 3.72 & 3.72 & 3.72 & 1.92 & 1.92 & 1.92 \\
\hline & CD @ 0.01 & 4.12 & 4.12 & 4.12 & 4.26 & 4.26 & 4.26 & 4.94 & 4.94 & 4.94 & 2.55 & 2.55 & 2.55 \\
\hline
\end{tabular}

Note: MP: Mid parent; BP: Better parent; SC1: GC-3 and SC2: GDVC-2

$*$ Significant at 5 per cent level and **Significant at 1 per cent level 
Table.2 Extent of mid parent heterosis, heterobeltiosis and standard heterosis in per cent for pods per plant in different environments and pooled over environments in cowpea

\begin{tabular}{|c|c|c|c|c|c|c|c|c|c|c|c|c|c|}
\hline Sr. & \multirow[t]{2}{*}{ Crosses } & \multicolumn{3}{|c|}{ Navsari } & \multicolumn{3}{|c|}{ Mangrol } & \multicolumn{3}{|c|}{ Achhalia } & \multicolumn{3}{|c|}{ Pooled } \\
\hline no. & & BP & SC1 & $\mathrm{SC2}$ & BP & SC1 & SC2 & BP & SC1 & SC2 & BP & SC1 & SC2 \\
\hline \multicolumn{14}{|c|}{ Direct Crosses } \\
\hline 1 & NCK-15-9 x NCK-15-10 & -8.23 & -13.16 & -9.59 & -0.06 & -2.51 & -6.48 & $-26.12 * *$ & $-13.86^{*}$ & $-20.78 * *$ & $-12.50 * *$ & $-9.90 * *$ & $-12.53 * *$ \\
\hline 2 & NCK-15-9 x NC-15-41 & 13.75 & -5.76 & -1.88 & $-25.64 * *$ & $-32.29 * *$ & $-35.05 * *$ & $-26.65 * *$ & -13.59 & $-20.53 * *$ & $-14.82 * *$ & $-17.11 * *$ & $-19.54 * *$ \\
\hline 3 & NCK-15-9 x NC-15-42 & $23.43^{*}$ & $-15.68^{*}$ & -12.21 & -3.97 & $-28.44 * *$ & $-31.35 * *$ & 5.67 & -2.4 & -10.24 & $10.59 *$ & $-15.41 * *$ & $-17.88 * *$ \\
\hline 4 & NCK-15-9 x NC-15-44 & 11.16 & -8.83 & -5.08 & 8.29 & $-13.13 *$ & $-16.67 * *$ & 8.29 & -4.48 & -12.15 & $9.23 *$ & $-8.78 *$ & $-11.44 * *$ \\
\hline 5 & NCK-15-9 x NC-15-45 & 9.91 & $14.36^{*}$ & $19.06 * *$ & $-22.66 * *$ & $-21.45 * *$ & $-24.65 * *$ & 0.05 & 6.71 & -1.86 & -3.93 & 0.02 & -2.91 \\
\hline 6 & NCK-15-10 x NC-15-41 & $27.81 * *$ & $20.94 * *$ & $25.91 * *$ & 5.18 & 2.59 & -1.59 & $12.56^{*}$ & $32.59 * *$ & $21.94 * *$ & $15.40 * *$ & $18.83 * *$ & $15.35 * *$ \\
\hline 7 & NCK-15-10 x NC-15-42 & -3.77 & -8.94 & -5.19 & $18.80 * *$ & $15.88 * *$ & $11.16^{*}$ & 1.12 & $17.90^{*}$ & 8.43 & 5.12 & $8.25 *$ & 5.08 \\
\hline 8 & NCK-15-10 x NC-15-44 & 6.08 & 0.38 & 4.51 & -9.16 & -11.4 & $-15.01 * *$ & -10.21 & 4.69 & -3.71 & -4.87 & -2.04 & -4.9 \\
\hline 9 & NCK-15-10 x NC-15-45 & $30.08 * *$ & $35.36 * *$ & $40.92 * *$ & $22.39 * *$ & $24.30 * *$ & $19.24 * *$ & $13.16^{*}$ & $31.93 * *$ & $21.34 * *$ & $25.41 * *$ & $30.57 * *$ & $26.76 * *$ \\
\hline 10 & NC-15-41 x NC-15-42 & $25.13 * *$ & 3.67 & 7.93 & $14.05^{*}$ & 3.85 & -0.38 & 2.41 & $20.63 * *$ & 10.94 & $12.47 * *$ & $9.44 * *$ & 6.24 \\
\hline 11 & NC-15-41 x NC-15-44 & 1.31 & $-16.06^{*}$ & -12.61 & $-25.83 * *$ & $-32.46^{* *}$ & $-35.21 * *$ & $-19.46 * *$ & -5.13 & $-12.75^{*}$ & $-15.50 * *$ & $-17.77 * *$ & $-20.18 * *$ \\
\hline 12 & NC-15-41 x NC-15-45 & 9.11 & $13.54^{*}$ & $18.21 *$ & -4.07 & -2.57 & -6.54 & 8.39 & $27.67 * *$ & $17.42 * *$ & $8.54 *$ & $13.00 * *$ & $9.70 * *$ \\
\hline 13 & NC-15-42 x NC-15-44 & 7.69 & -11.68 & -8.05 & -6.55 & $-25.03 * *$ & $-28.08 * *$ & -3.31 & -10.7 & $-17.87 * *$ & 0.9 & $-15.74 * *$ & $-18.20 * *$ \\
\hline 14 & NC-15-42 x NC-15-45 & $14.59^{*}$ & $19.24 * *$ & $24.14 * *$ & 5.94 & 7.6 & 3.22 & 11.41 & $18.83 * *$ & 9.29 & $10.72 * *$ & $15.28 * *$ & $11.91 * *$ \\
\hline 15 & NC-15-44 x NC-15-45 & -1.11 & 2.91 & 7.13 & 5.34 & 6.98 & 2.63 & -3.02 & 3.44 & -4.87 & 0.3 & 4.43 & 1.37 \\
\hline
\end{tabular}


Int.J.Curr.Microbiol.App.Sci (2018) 7(7): 3400-3413

Table.2 Contd......

\begin{tabular}{|c|c|c|c|c|c|c|c|c|c|c|c|c|c|}
\hline Sr. & \multirow[t]{2}{*}{ Crosses } & \multicolumn{3}{|c|}{ Navsari } & \multicolumn{3}{|c|}{ Mangrol } & \multicolumn{3}{|c|}{ Achhalia } & \multicolumn{3}{|c|}{ Pooled } \\
\hline no. & & BP & SC1 & SC2 & BP & SC1 & SC2 & BP & SC1 & $\mathrm{SC2}$ & $\mathbf{B P}$ & SC1 & SC2 \\
\hline \multicolumn{14}{|c|}{ Reciprocal Crosses } \\
\hline 16 & NCK-15-10 x NCK-15-9 & $16.40^{*}$ & 10.14 & $14.67 *$ & -8.19 & -10.45 & $-14.09 * *$ & $-16.01 * *$ & -2.07 & -9.94 & -3.6 & -0.73 & -3.64 \\
\hline 17 & NC-15-41 x NCK-15-9 & 9.78 & -9.05 & -5.31 & $-18.28 * *$ & $-25.59 * *$ & $-28.62 * *$ & $-18.86 * *$ & -4.42 & -12.1 & $-10.51 * *$ & $-12.93 * *$ & $-15.47 * *$ \\
\hline 18 & NC-15-41 x NCK-15-10 & $28.59 * *$ & $21.68 * *$ & $26.68 * *$ & $22.20 * *$ & $19.20 * *$ & $14.34 * *$ & $12.47 *$ & $32.48 * *$ & $21.84 * *$ & $20.90 * *$ & $24.50 * *$ & $20.86 * *$ \\
\hline 19 & NC-15-42 x NCK-15-9 & $23.43^{*}$ & $-15.68 *$ & -12.21 & 5.02 & $-21.73 * *$ & $-24.92 * *$ & 7.33 & -0.87 & -8.84 & $14.14 * *$ & $-12.69 * *$ & $-15.24 * *$ \\
\hline 20 & NC-15-42 x NCK-15-10 & 4.35 & -1.26 & 2.8 & 3.25 & 0.72 & -3.39 & -6.09 & 9.5 & 0.7 & 0.03 & 3.01 & 0.01 \\
\hline 21 & NC-15-42 x NC-15-41 & 11.9 & -7.29 & -3.48 & $-20.31 * *$ & $-27.43 * *$ & $-30.39 * *$ & 1.99 & $20.14 * *$ & 10.49 & -2.04 & -4.68 & -7.47 \\
\hline 22 & NC-15-44 x NCK-15-9 & 15.17 & -5.54 & -1.66 & -2.86 & $-22.07 * *$ & $-25.24 * *$ & $16.96^{*}$ & 3.17 & -5.12 & $10.11^{*}$ & $-8.04 *$ & $-10.73 * *$ \\
\hline 23 & NC-15-44 x NCK-15-10 & $23.68 * *$ & $17.04^{*}$ & $21.85 * *$ & 7.26 & 4.63 & 0.36 & 3.75 & $20.96 * *$ & 11.24 & $10.98 * *$ & $14.28 * *$ & $10.94 * *$ \\
\hline 24 & NC-15-44 x NC-15-41 & $29.12 * *$ & 6.97 & 11.37 & $16.63 * *$ & 6.20 & 1.88 & 0.23 & $18.07 *$ & 8.58 & $13.51 * *$ & $10.45^{* *}$ & 7.22 \\
\hline 25 & NC-15-44 x NC-15-42 & 15.74 & -5.08 & -1.18 & 12.73 & -9.56 & $-13.25 *$ & $17.61 *$ & 8.62 & -0.1 & $17.42 * *$ & -1.94 & -4.81 \\
\hline 26 & NC-15-45 x NCK-15-9 & $13.07 *$ & $17.65^{*}$ & $22.49 * *$ & 10.34 & $12.07 *$ & 7.50 & 1.94 & 8.73 & 0.01 & $8.36^{*}$ & $12.82 * *$ & $9.52 * *$ \\
\hline 27 & NC-15-45 x NCK-15-10 & $29.56 * *$ & $34.81 * *$ & $40.35 * *$ & $11.39 *$ & $13.13^{*}$ & 8.52 & $25.28 * *$ & $46.07 * *$ & $34.34 * *$ & $26.28 * *$ & $31.47 * *$ & $27.63 * *$ \\
\hline 28 & NC-15-45 x NC-15-41 & $22.81 * *$ & $27.80 * *$ & $33.05 * *$ & 9.5 & $11.22 *$ & 6.69 & $16.13 * *$ & $36.79 * *$ & $25.80 * *$ & $20.42 * *$ & $25.37 * *$ & $21.71 * *$ \\
\hline 29 & NC-15-45 x NC-15-42 & 8.15 & 12.53 & $17.16^{*}$ & 5.94 & 7.6 & 3.22 & 4.66 & 11.63 & 2.66 & 6.24 & $10.61 * *$ & 7.37 \\
\hline \multirow[t]{4}{*}{30} & NC-15-45 x NC-15-44 & 5.95 & 10.25 & $14.78^{*}$ & $19.64 * *$ & $21.51 * *$ & $16.56 * *$ & 3.53 & 10.43 & 1.56 & $9.51 * *$ & $14.01 * *$ & $10.68 * *$ \\
\hline & S.E.(d) \pm & 1.79 & 1.79 & 1.79 & 1.62 & 1.62 & 1.62 & 2.11 & 2.11 & 2.11 & 1.06 & 1.06 & 1.06 \\
\hline & CD@ 0.05 & 3.57 & 3.57 & 3.57 & 3.23 & 3.23 & 3.23 & 4.22 & 4.22 & 4.22 & 2.12 & 2.12 & 2.12 \\
\hline & CD @ 0.01 & 4.74 & 4.74 & 4.74 & 4.29 & 4.29 & 4.29 & 5.6 & 5.6 & 5.6 & 2.81 & 2.81 & 2.81 \\
\hline
\end{tabular}


Table.3 Extent of mid parent heterosis, heterobeltiosis and standard heterosis in per cent for green seed weight (g) in different environments and pooled over environments in cowpea

\begin{tabular}{|c|c|c|c|c|c|c|c|c|c|c|c|c|c|}
\hline Sr. & \multirow[t]{2}{*}{ Crosses } & \multicolumn{3}{|c|}{ Navsari } & \multicolumn{3}{|c|}{ Mangrol } & \multicolumn{3}{|c|}{ Achhalia } & \multicolumn{3}{|c|}{ Pooled } \\
\hline no. & & BP & SC1 & SC2 & BP & SC1 & SC2 & BP & SC1 & SC2 & BP & SC1 & SC2 \\
\hline \multicolumn{14}{|c|}{ Direct Crosses } \\
\hline 1 & NCK-15-9 x NCK-15-10 & -2.4 & -8.49 & $-11.85 * *$ & $15.91 * *$ & $17.61 * *$ & $11.46^{*}$ & $-13.26 * *$ & $-18.71 * *$ & $-22.49 * *$ & -0.35 & $-6.58 * *$ & $-10.79 * *$ \\
\hline 2 & NCK-15-9 x NC-15-41 & $-11.56 * *$ & -3.46 & -7 & $13.78 * *$ & $14.28 * *$ & 8.3 & -0.15 & $-14.51 * *$ & $-18.48 * *$ & 0.7 & -0.78 & -5.25 \\
\hline 3 & NCK-15-9 x NC-15-42 & -0.82 & -7.01 & $-10.42 *$ & -3.45 & $16.34 * *$ & $10.25^{*}$ & $-28.02 * *$ & $-18.45 * *$ & $-22.24 * *$ & -4.14 & -4.40 & $-8.71 * *$ \\
\hline 4 & NCK-15-9 x NC-15-44 & $-14.28 * *$ & -2.33 & -5.92 & $-11.21 * *$ & 7.31 & 1.70 & $-15.97 * *$ & $-7.16^{*}$ & $-11.48 * *$ & $-11.32 * *$ & 1.78 & -2.81 \\
\hline 5 & NCK-15-9 x NC-15-45 & -5.46 & -8.51 & $-11.86 * *$ & 1.86 & 5.52 & 0.01 & -0.36 & -1.00 & -5.60 & $-5.82 *$ & -0.96 & -5.42 \\
\hline 6 & NCK-15-10 x NC-15-41 & 1.11 & $10.38^{*}$ & 6.33 & $9.92 *$ & $13.87 * *$ & 7.91 & -1.14 & $-7.35^{*}$ & $-11.66 * *$ & 4.20 & 2.66 & -1.97 \\
\hline 7 & NCK-15-10 x NC-15-42 & $12.74 *$ & -0.33 & -3.99 & -6.06 & $13.19^{*}$ & 7.27 & $-6.86^{*}$ & 5.53 & 0.62 & $6.42 *$ & $6.13 *$ & 1.35 \\
\hline 8 & NCK-15-10 x NC-15-44 & $-10.11 *$ & 2.42 & -1.34 & 0.15 & $21.04 * *$ & $14.71 * *$ & -4.48 & 5.53 & 0.62 & $-6.89 * *$ & $6.86^{* *}$ & 2.04 \\
\hline 9 & NCK-15-10 x NC-15-45 & 8.69 & 5.19 & 1.33 & -5.00 & -3.60 & -8.65 & -0.99 & -1.63 & -6.20 & 2.44 & $7.73 * *$ & 2.87 \\
\hline 10 & NC-15-41 x NC-15-42 & -0.46 & 8.67 & 4.68 & -1.52 & $18.67 * *$ & $12.46^{*}$ & $-20.60 * *$ & $-10.04 * *$ & $-14.23 * *$ & -1.43 & -1.70 & -6.13 \\
\hline 11 & NC-15-41 x NC-15-44 & $-10.69 * *$ & 1.77 & -1.97 & -0.78 & $19.91 * *$ & $13.64 * *$ & $-22.65 * *$ & $-14.54 * *$ & $-18.52 * *$ & $-11.73 * *$ & 1.3 & -3.26 \\
\hline 12 & NC-15-41 x NC-15-45 & 1.69 & $11.01 *$ & 6.94 & 5.45 & $27.07 * *$ & $20.42 * *$ & 5.88 & 5.2 & 0.31 & $6.25 * *$ & $11.74 * *$ & $6.71 * *$ \\
\hline 13 & NC-15-42 x NC-15-44 & -4.48 & 8.83 & 4.84 & $-20.24 * *$ & -3.6 & -8.65 & $-8.26 * *$ & 3.93 & -0.9 & -1.73 & $12.78 * *$ & $7.70 * *$ \\
\hline 14 & NC-15-42 x NC-15-45 & -4.96 & -8.03 & $-11.40 * *$ & -5.08 & $14.72 * *$ & 8.71 & -2.67 & $10.27 * *$ & 5.14 & $-5.14 *$ & -0.24 & -4.73 \\
\hline 15 & NC-15-44 x NC-15-45 & -3.96 & $9.43 *$ & 5.41 & $-10.23 *$ & $14.72 * *$ & 8.71 & -3.76 & 6.33 & 1.38 & -4.17 & $9.99 * *$ & 5.03 \\
\hline
\end{tabular}


Int.J.Curr.Microbiol.App.Sci (2018) 7(7): 3400-3413

Table.3 Contd....

\begin{tabular}{|c|c|c|c|c|c|c|c|c|c|c|c|c|c|}
\hline \multirow{2}{*}{$\begin{array}{l}\text { Sr. } \\
\text { no. }\end{array}$} & \multirow[t]{2}{*}{ Crosses } & \multicolumn{3}{|c|}{ Navsari } & \multicolumn{3}{|c|}{ Mangrol } & \multicolumn{3}{|c|}{ Achhalia } & \multicolumn{3}{|c|}{ Pooled } \\
\hline & & BP & SC1 & SC2 & BP & SC1 & SC2 & $\mathbf{B P}$ & SC1 & SC2 & $\mathbf{B P}$ & SC1 & SC2 \\
\hline \multicolumn{14}{|c|}{ Reciprocal Crosses } \\
\hline 16 & NCK-15-10 x NCK-15-9 & 4.5 & -2.02 & -5.61 & $15.67 * *$ & $19.83 * *$ & $13.56^{* *}$ & -4.41 & $\begin{array}{c}- \\
10.41 * *\end{array}$ & $\begin{array}{c}- \\
14.58 * *\end{array}$ & $8.63 * *$ & 1.83 & -2.76 \\
\hline 17 & NC-15-41 x NCK-15-9 & -3.15 & 5.74 & 1.85 & $17.31 * *$ & $19.03 * *$ & $12.80 * *$ & $25.33 * *$ & $7.31 *$ & 2.31 & $12.07 * *$ & $10.42 * *$ & 5.44 \\
\hline 18 & NC-15-41 x NCK-15-10 & -4.05 & 4.75 & 0.9 & 3.8 & 7.53 & 1.9 & $12.60 * *$ & 5.53 & 0.62 & $7.47 * *$ & $5.89 *$ & 1.12 \\
\hline 19 & NC-15-42 x NCK-15-9 & $16.43 * *$ & $9.17 *$ & 5.16 & $27.60 * *$ & $28.16^{* *}$ & $21.45^{* *}$ & $\begin{array}{c}- \\
23.97 * *\end{array}$ & $\overline{-}^{-}$ & $\begin{array}{c}- \\
17.87 * *\end{array}$ & $7.29 * *$ & $7.00 * *$ & 2.18 \\
\hline 20 & NC-15-42 x NCK-15-10 & 0.19 & $-11.43 *$ & $\begin{array}{c}- \\
14.68 * *\end{array}$ & $13.25 * *$ & $17.32 * *$ & $11.18^{*}$ & 0.29 & $13.62 * *$ & $8.33 *$ & $6.58 * *$ & $6.28 *$ & 1.5 \\
\hline 21 & NC-15-42 x NC-15-41 & $\overline{-}^{-}$ & -6.94 & $-10.35^{*}$ & $25.94 * *$ & $27.80 * *$ & $21.11 * *$ & -4.93 & $7.71 *$ & 2.7 & $9.29 * *$ & $8.99 * *$ & 4.08 \\
\hline 22 & NC-15-44 x NCK-15-9 & 3.16 & $17.54 * *$ & $13.23 * *$ & -1.52 & $18.67 * *$ & $12.46^{*}$ & -0.97 & $9.41 * *$ & 4.32 & 0.24 & $15.04 * *$ & $9.86^{* *}$ \\
\hline 23 & NC-15-44 x NCK-15-10 & $9.02 *$ & $24.23 * *$ & $19.67 * *$ & $\begin{array}{c}- \\
11.27 * *\end{array}$ & 6.92 & 1.32 & 5.47 & $16.52 * *$ & $11.10^{* *}$ & 1.2 & $16.15 * *$ & $10.91 * *$ \\
\hline 24 & NC-15-44 x NC-15-41 & 2.86 & $17.21 * *$ & $12.90 * *$ & 6.36 & $28.16^{* *}$ & $21.45 * *$ & -2.35 & $7.89 *$ & 2.87 & 2.25 & $17.35 * *$ & $12.06^{* *}$ \\
\hline 25 & NC-15-44 x NC-15-42 & -0.23 & $13.68 * *$ & $9.51 *$ & $23.45 * *$ & $48.76^{* *}$ & $40.97 * *$ & $\begin{array}{c}- \\
16.03 * *\end{array}$ & -4.86 & $-9.29 * *$ & 2.89 & $18.08 * *$ & $12.76^{* *}$ \\
\hline 26 & NC-15-45 x NCK-15-9 & 3.18 & -0.14 & -3.81 & 0.05 & $20.92 * *$ & $14.59 * *$ & -5.85 & -6.45 & $\begin{array}{c}- \\
10.80^{* *}\end{array}$ & -0.93 & 4.19 & -0.5 \\
\hline 27 & NC-15-45 x NCK-15-10 & 0.96 & -2.29 & -5.88 & -0.99 & $19.66 * *$ & $13.40 * *$ & $7.60 *$ & $6.91 *$ & 1.94 & 2.46 & $7.75^{* *}$ & 2.9 \\
\hline 28 & NC-15-45 x NC-15-41 & 2.42 & $11.81 * *$ & 7.71 & -0.14 & $20.69 * *$ & $14.37 * *$ & $14.03 * *$ & $13.30 * *$ & $8.02 *$ & $9.44 * *$ & $15.09 * *$ & $9.91 * *$ \\
\hline 29 & NC-15-45 x NC-15-42 & 3.34 & 0.01 & -3.66 & 0.72 & $21.73 * *$ & $15.36^{* *}$ & $\begin{array}{c}- \\
20.95 * *\end{array}$ & $\begin{array}{c}- \\
10.44 * *\end{array}$ & $\overline{-}^{-}$ & -1.97 & 3.1 & -1.55 \\
\hline \multirow[t]{4}{*}{30} & NC-15-45 x NC-15-44 & $-9.41 *$ & 3.22 & -0.57 & $11.21 * *$ & 7.31 & 1.7 & $6.77 *$ & $17.97 * *$ & $12.48 * *$ & $-4.45^{*}$ & $9.66 * *$ & 4.72 \\
\hline & S.E.(d) \pm & 0.89 & 0.89 & 0.89 & 0.92 & 0.92 & 0.92 & 0.68 & 0.68 & 0.68 & 0.48 & 0.48 & 0.48 \\
\hline & CD @ 0.05 & 1.77 & 1.77 & 1.77 & 1.83 & 1.83 & 1.83 & 1.36 & 1.36 & 1.36 & 0.96 & 0.96 & 0.96 \\
\hline & CD @ 0.01 & 2.35 & 2.35 & 2.35 & 2.43 & 2.43 & 2.43 & 1.81 & 1.81 & 1.81 & 1.28 & 1.28 & 1.28 \\
\hline
\end{tabular}


Table.4 Extent of mid parent heterosis, heterobeltiosis and standard heterosis in per cent for green pod yield per plant ( $\mathrm{g}$ ) in different environments and pooled over environments in cowpea

\begin{tabular}{|c|c|c|c|c|c|c|c|c|c|c|c|c|c|}
\hline Sr. & \multirow[t]{2}{*}{ Crosses } & \multicolumn{3}{|c|}{ Navsari } & \multicolumn{3}{|c|}{ Mangrol } & \multicolumn{3}{|c|}{ Achhalia } & \multicolumn{3}{|c|}{ Pooled } \\
\hline no. & & BP & SC1 & SC2 & BP & SC1 & SC2 & BP & SC1 & SC2 & BP & SC1 & $\mathrm{SC2}$ \\
\hline \multicolumn{14}{|c|}{ Direct Crosses } \\
\hline 1 & NCK-15-9 x NCK-15-10 & -13.33 & $-16.99 *$ & $-23.21 * *$ & -0.05 & -2.21 & -4.17 & $-17.33 *$ & -16.36 & $-21.24 * *$ & $-10.47 *$ & $-11.94 *$ & $-16.49 * *$ \\
\hline 2 & NCK-15-9 x NC-15-41 & 2.05 & -6.78 & -13.76 & -16.45 & $\begin{array}{c}- \\
2.33 \mathrm{E}+10\end{array}$ & $-24.93 * *$ & $-17.96^{*}$ & -16.11 & $-21.01 * *$ & $-11.36^{*}$ & $-15.51 * *$ & $-19.88 * *$ \\
\hline 3 & NCK-15-9 x NC-15-42 & 13.69 & -14.43 & $-20.84 * *$ & 5.6 & $-19.73^{*}$ & $-21.35 * *$ & 10.1 & -6.07 & -11.56 & $12.39 *$ & $-13.21 * *$ & $-17.69 * *$ \\
\hline 4 & NCK-15-9 x NC-15-44 & $23.89 *$ & 3.2 & -4.53 & 3.27 & -12.28 & -14.04 & 18.05 & -3.69 & -9.31 & $14.99 * *$ & -4.3 & $-9.24 *$ \\
\hline 5 & NCK-15-9 x NC-15-45 & 7.64 & 13.12 & 4.65 & -3.83 & 1.02 & -1 & -2.93 & -4.42 & -10.01 & 0.22 & 2.96 & -2.36 \\
\hline 6 & NCK-15-10 x NC-15-41 & $24.92 * *$ & $19.63 *$ & 10.67 & 12.12 & 9.7 & 7.5 & 8.51 & 10.95 & 4.47 & $15.21 * *$ & $13.32 * *$ & 7.46 \\
\hline 7 & NCK-15-10 x NC-15-42 & 7.46 & 2.91 & -4.8 & $17.78^{*}$ & 15.24 & 12.93 & 10.84 & 12.14 & 5.59 & $12.04 *$ & $10.21 *$ & 4.51 \\
\hline 8 & NCK-15-10 x NC-15-44 & 3.68 & -0.7 & -8.14 & -8.67 & -10.64 & -12.43 & -0.87 & 0.29 & -5.56 & -1.99 & -3.6 & -8.58 \\
\hline 9 & NCK-15-10 x NC-15-45 & $27.40 * *$ & $33.89 * *$ & $23.86 * *$ & $21.68 * *$ & $27.83 * *$ & $25.26 * *$ & $23.28 * *$ & $24.73 * *$ & $17.45^{*}$ & $25.24 * *$ & $28.67 * *$ & $22.02 * *$ \\
\hline 10 & NC-15-41 x NC-15-42 & $26.90 * *$ & $15.93 *$ & 7.24 & 15.16 & 5.6 & 3.48 & 12.07 & 14.59 & 7.9 & $17.58 * *$ & $12.08^{*}$ & 6.29 \\
\hline 11 & NC-15-41 x NC-15-44 & -9.11 & $-16.97 *$ & $-23.19 * *$ & -12.77 & $-20.02 *$ & $-21.62 * *$ & -10.54 & -8.52 & -13.86 & $-10.80 *$ & $-14.98 * *$ & $-19.37 * *$ \\
\hline 12 & NC-15-41 x NC-15-45 & 6.86 & 12.31 & 3.9 & -6.96 & -2.26 & -4.22 & 8.67 & 11.12 & 4.63 & 4.27 & 7.13 & 1.59 \\
\hline 13 & NC-15-42 x NC-15-44 & 5.45 & -12.17 & $-18.75^{*}$ & -3.79 & $-18.27 *$ & $-19.91 *$ & 1.38 & -13.52 & $-18.57 *$ & 2.56 & $-14.64 * *$ & $-19.05^{* *}$ \\
\hline 14 & NC-15-42 x NC-15-45 & -9.39 & -4.77 & -11.9 & 2.22 & 7.39 & 5.23 & 14.74 & 12.98 & 6.38 & 2.67 & 5.48 & 0.03 \\
\hline 15 & NC-15-44 x NC-15-45 & -3.15 & 1.79 & -5.84 & 1.66 & 6.8 & 4.66 & 5.69 & 4.06 & -2.01 & 1.46 & 4.23 & -1.16 \\
\hline
\end{tabular}


Int.J.Curr.Microbiol.App.Sci (2018) 7(7): 3400-3413

Table.4 Contd......

\begin{tabular}{|c|c|c|c|c|c|c|c|c|c|c|c|c|c|}
\hline \multirow{2}{*}{$\begin{array}{l}\text { Sr. } \\
\text { no. }\end{array}$} & \multirow[t]{2}{*}{ Crosses } & \multicolumn{3}{|c|}{ Navsari } & \multicolumn{3}{|c|}{ Mangrol } & \multicolumn{3}{|c|}{ Achhalia } & \multicolumn{3}{|c|}{ Pooled } \\
\hline & & BP & SC1 & SC2 & BP & SC1 & SC2 & BP & SC1 & $\mathrm{SC2}$ & BP & SC1 & SC2 \\
\hline \multicolumn{14}{|c|}{ Reciprocal Crosses } \\
\hline 16 & NCK-15-10 x NCK-15-9 & 13.76 & 8.95 & 0.79 & -7.75 & -9.73 & -11.55 & -6.87 & -5.78 & -11.28 & -0.74 & -2.37 & -7.41 \\
\hline 17 & NC-15-41 x NCK-15-9 & -1.52 & -10.03 & $-16.77 *$ & $-17.23 *$ & $-24.10 * *$ & $-25.62 * *$ & -9.91 & -7.88 & -13.26 & -9.64 & $-13.87 * *$ & $\begin{array}{c}- \\
18.32 * *\end{array}$ \\
\hline 18 & NC-15-41 x NCK-15-10 & $36.62 * *$ & $30.84 * *$ & $21.04 * *$ & $21.00 * *$ & $18.39^{*}$ & $16.01^{*}$ & $22.46^{* *}$ & $25.22 * *$ & $17.91^{*}$ & $26.86^{* *}$ & $24.78 * *$ & $18.33 * *$ \\
\hline 19 & NC-15-42 x NCK-15-9 & 10.81 & $-16.59 *$ & $-22.84 * *$ & 13.51 & -13.73 & $-15.46^{*}$ & 11.71 & -4.7 & -10.27 & $14.66^{*}$ & $-11.45^{*}$ & $\stackrel{-}{16.03 * *}$ \\
\hline 20 & NC-15-42 x NCK-15-10 & 1.98 & -2.33 & -9.65 & 3.08 & 0.86 & -1.17 & 3.39 & 4.6 & -1.51 & 2.85 & 1.16 & -4.07 \\
\hline 21 & NC-15-42 x NC-15-41 & 0.38 & -8.3 & $-15.17 *$ & $-19.13^{*}$ & $-25.85 * *$ & $-27.34 * *$ & 11.64 & 14.15 & 7.49 & -1.48 & -6.09 & $-10.95 *$ \\
\hline 22 & NC-15-44 x NCK-15-9 & 12.17 & -6.56 & -13.56 & -6.72 & $-20.76^{* *}$ & $-22.35 * *$ & $21.25^{*}$ & -1.08 & -6.86 & 9.02 & $-9.26^{*}$ & $\begin{array}{c}- \\
13.95 * *\end{array}$ \\
\hline 23 & NC-15-44 x NCK-15-10 & $20.88^{*}$ & $15.77 *$ & 7.1 & 7.59 & 5.27 & 3.16 & 13.55 & 14.89 & 8.18 & $13.89 * *$ & $12.03 *$ & 6.24 \\
\hline 24 & NC-15-44 x NC-15-41 & $38.78 * *$ & $26.78 * *$ & $17.28^{*}$ & 15.66 & 6.06 & 3.93 & $28.02 * *$ & $30.90 * *$ & $23.26 * *$ & $27.43^{* *}$ & $21.47 * *$ & $15.19 * *$ \\
\hline 25 & NC-15-44 x NC-15-42 & -2.28 & $-18.60^{*}$ & $-24.70 * *$ & 7.25 & -8.9 & -10.73 & 6.39 & -9.24 & -14.54 & 5.59 & $-12.12 *$ & $\begin{array}{c}- \\
16.66^{* *}\end{array}$ \\
\hline 26 & NC-15-45 x NCK-15-9 & 2.48 & 7.7 & -0.37 & 6.26 & 11.63 & 9.38 & 2.22 & 0.65 & -5.23 & 3.65 & 6.49 & 0.99 \\
\hline 27 & NC-15-45 x NCK-15-10 & $23.10 * *$ & $29.37 * *$ & $19.68 * *$ & $28.74 * *$ & $35.25^{* *}$ & $32.53 * *$ & 13.23 & 14.56 & 7.87 & $22.70^{* *}$ & $26.05^{* *}$ & $19.54 * *$ \\
\hline 28 & NC-15-45 x NC-15-41 & $25.44 * *$ & $31.83^{*}$ & $21.96^{* *}$ & $26.68 * *$ & $33.08 * *$ & $30.41 * *$ & $26.25^{* *}$ & $29.09 * *$ & $21.55^{* *}$ & $27.77 * *$ & $31.27 * *$ & $24.48 * *$ \\
\hline 29 & NC-15-45 x NC-15-42 & -2.2 & 2.79 & -4.91 & 2.22 & 7.39 & 5.23 & 8.17 & 6.51 & 0.29 & 2.79 & 5.61 & 0.15 \\
\hline \multirow[t]{4}{*}{30} & NC-15-45 x NC-15-44 & 3.77 & 9.06 & 0.89 & $14.78^{*}$ & $20.58 * *$ & $18.16^{*}$ & 7.08 & 5.44 & -0.72 & 8.57 & $11.54^{*}$ & 5.78 \\
\hline & S.E.(d) \pm & 7.28 & 7.28 & 7.28 & 7.3 & 7.3 & 7.3 & 8.52 & 8.52 & 8.52 & 4.46 & 4.46 & 4.46 \\
\hline & CD @ 0.05 & 14.52 & 14.52 & 14.52 & 14.56 & 14.56 & 14.56 & 16.99 & 16.99 & 16.99 & 8.89 & 8.89 & 8.89 \\
\hline & CD @ 0.01 & 19.27 & 19.27 & 19.27 & 19.33 & 19.33 & 19.33 & 22.56 & 22.56 & 22.56 & 11.8 & 11.8 & 11.8 \\
\hline
\end{tabular}


Table.5 Magnitude of heterobeltiosis and standard heterosis in cowpea on pooled basis

\begin{tabular}{|c|c|c|c|c|c|c|c|c|c|c|c|}
\hline \multirow[t]{3}{*}{ Characters } & \multicolumn{6}{|c|}{ Range of heterosis (per cent) } & \multicolumn{3}{|c|}{$\begin{array}{l}\text { Number of crosses with significant } \\
\text { heterosis in desirable direction }\end{array}$} & \multicolumn{2}{|c|}{ Best crosse } \\
\hline & \multicolumn{2}{|c|}{ Heterobeltiosis } & \multicolumn{2}{|c|}{$\begin{array}{c}\text { Standard } \\
\text { heterosis } \\
\text { over Check- } \\
\text { GC-3 }\end{array}$} & \multicolumn{2}{|c|}{$\begin{array}{c}\text { Standard } \\
\text { heterosis } \\
\text { over Check- } \\
\text { GDVC-2 }\end{array}$} & \multirow[t]{2}{*}{ Heterobeltiosis } & \multirow{2}{*}{$\begin{array}{c}\text { Standard } \\
\text { heterosis } \\
\text { over } \\
\text { Check- } \\
\text { GC-3 }\end{array}$} & \multirow{2}{*}{$\begin{array}{c}\text { Standard } \\
\text { heterosis } \\
\text { over } \\
\text { Check- } \\
\text { GDVC-2 }\end{array}$} & \multirow[t]{2}{*}{ Heterobeltiosis } & \multirow[t]{2}{*}{$\begin{array}{c}\text { Standard } \\
\text { heterosis } \\
\text { over } \\
\text { Check- } \\
\text { GDVC-2 }\end{array}$} \\
\hline & From & To & From & To & From & To & & & & & \\
\hline $\begin{array}{l}\text { Days to 50per cent } \\
\text { flowering }\end{array}$ & -10.85 & 18.41 & -8.96 & 10.80 & 13.78 & 4.93 & 7 & 7 & 22 & NC-15-44 x NCK-15-9 & $\begin{array}{l}\text { NC-15-42 x } \\
\text { NC-15-44 }\end{array}$ \\
\hline Plant height $(\mathrm{cm})$ & -17.97 & 12.53 & -14.54 & 20.73 & -24.98 & 5.99 & 4 & 6 & 0 & NC-15-45 x NCK-15-9 & $\begin{array}{l}\text { NC-15-42 x } \\
\text { NCK-15-10 }\end{array}$ \\
\hline $\begin{array}{l}\text { Primary branches } \\
\text { per plant }\end{array}$ & -12.73 & 33.75 & -7.55 & 32.55 & -4.38 & 37.10 & 9 & 12 & 14 & $\begin{array}{l}\text { NCK-15-10 x NC-15- } \\
45\end{array}$ & $\begin{array}{l}\text { NCK-15-10 x } \\
\text { NC-15-45 }\end{array}$ \\
\hline Pods per plant & -15.50 & 26.28 & -17.77 & 30.57 & -20.18 & 27.63 & 17 & 14 & 10 & $\begin{array}{c}\text { NC-15-45 x NCK-15- } \\
10\end{array}$ & $\begin{array}{l}\text { NC-15-45 x } \\
\text { NCK-15-10 }\end{array}$ \\
\hline Pod length $(\mathrm{cm})$ & -14.40 & 14.09 & -17.15 & 13.11 & -16.76 & 13.65 & 4 & 5 & 7 & $\begin{array}{c}\text { NC-15-45 x NCK-15- } \\
10\end{array}$ & $\begin{array}{l}\text { NC-15-44 x } \\
\text { NC-15-45 }\end{array}$ \\
\hline Seeds per pod & -12.59 & 19.67 & -17.88 & 16.59 & -13.06 & 23.43 & 6 & 3 & 9 & $\begin{array}{c}\text { NC-15-45 x NCK-15- } \\
10\end{array}$ & $\begin{array}{l}\text { NC-15-45 x } \\
\text { NCK-15-10 }\end{array}$ \\
\hline $\begin{array}{l}\text { Green seed weight } \\
(\mathrm{g})\end{array}$ & -11.73 & 12.07 & -6.58 & 18.08 & -10.79 & 12.76 & 9 & 18 & 7 & NC-15-41 x NCK-15-9 & $\begin{array}{l}\text { NC-15-44 x } \\
\text { NC-15-42 }\end{array}$ \\
\hline $\begin{array}{l}\text { Green pod yield } \\
\text { per plant }(\mathrm{g})\end{array}$ & -11.36 & 27.77 & -15.51 & 31.27 & -19.88 & 24.48 & 14 & 13 & 9 & NC-15-45 x NC-15-41 & $\begin{array}{l}\text { NC-15-45 x } \\
\text { NC-15-41 }\end{array}$ \\
\hline $\begin{array}{l}\text { Shelling ratio (per } \\
\text { cent) }\end{array}$ & -10.70 & 10.71 & -5.72 & 12.04 & -12.42 & 5.03 & 6 & 10 & 3 & $\begin{array}{l}\text { NCK-15-10 x NC-15- } \\
41\end{array}$ & $\begin{array}{l}\text { NCK-15-10 x } \\
\text { NC-15-41 }\end{array}$ \\
\hline $\begin{array}{l}\text { Protein content } \\
\text { (per cent) }\end{array}$ & -16.50 & 12.92 & -15.31 & 15.02 & -13.60 & 17.35 & 12 & 13 & 16 & NC-15-41 x NC-15-45 & $\begin{array}{l}\text { NC-15-42 x } \\
\text { NC-15-44 }\end{array}$ \\
\hline
\end{tabular}


Table.6 Top ten crosses on the basis of per se performance with heterobeltiosis and standard heterosis for green pod yield per plant with yield attributing traits which registered significant and desirable heterobeltiosis in cowpea

\begin{tabular}{|c|c|c|c|c|c|c|}
\hline $\begin{array}{l}\text { Sr. } \\
\text { No. }\end{array}$ & Crosses & $\begin{array}{l}\text { Green pod } \\
\text { yield per } \\
\text { plant }(\mathrm{g})\end{array}$ & $\begin{array}{c}\text { Better } \\
\text { parent } \\
\text { heterosis } \\
\text { (per cent) }\end{array}$ & $\begin{array}{l}\text { Standard } \\
\text { heterosis } \\
\text { over GC-3 }\end{array}$ & $\begin{array}{l}\text { Standard } \\
\text { heterosis } \\
\text { over } \\
\text { GDVC-2 }\end{array}$ & $\begin{array}{l}\text { Other characters which registered significant and } \\
\text { desirable standard heterosis }\end{array}$ \\
\hline 1. & NC-15-45 X NC-15-41 & 126.30 & $27.77 * *$ & $31.27 * *$ & $24.48 * *$ & DF, PB, PPP,PL,SPP,GSW,PRT \\
\hline 2. & $\begin{array}{l}\text { NCK-15-10 X NC-15- } \\
45\end{array}$ & 123.79 & $25.24 * *$ & $28.67 * *$ & $22.02 * *$ & PB,PPP,PL,GSW,SP, \\
\hline 3 & $\begin{array}{l}\text { NC-15-45 X NCK-15- } \\
10\end{array}$ & 121.28 & $22.70 * *$ & $26.05 * *$ & $19.54 * *$ & PB, PPP,PL,SPP,GSW,PRT \\
\hline 4 & $\begin{array}{l}\text { NC-15-41 X NCK-15- } \\
10\end{array}$ & 120.06 & $26.86 * *$ & $24.78 * *$ & $18.33 * *$ & DF, PB,PPP,SPP,GSW \\
\hline 5 & NC-15-44 X NC-15-41 & 116.86 & $27.43 * *$ & $21.47 * *$ & $15.19 * *$ & DF, PB, PPP,PL,SPP,GSW \\
\hline 6 & $\begin{array}{c}\text { NCK-15-10 X NC-15- } \\
41\end{array}$ & 109.02 & $15.21 * *$ & $13.32 * *$ & $7.46^{* *}$ & DF, PB,DM,PPP,GSW \\
\hline 7 & NC-15-41 X NC-15-42 & 107.83 & $17.58 * *$ & $12.08 * *$ & $6.29 * *$ & DF, PPP,PL \\
\hline 8 & $\begin{array}{l}\text { NC-15-44 X NCK-15- } \\
10\end{array}$ & 107.78 & $13.89 * *$ & $12.03 * *$ & $6.24 * *$ & DF,PPP \\
\hline 9 & NC-15-45 X NC-15-44 & 107.32 & 8.57 & $11.54 * *$ & $5.78 * *$ & DF, PB, PPP,PL,SPP \\
\hline 10 & $\begin{array}{l}\text { NCK-15-10 X NC-15- } \\
42\end{array}$ & 106.03 & $12.04 * *$ & $10.21 * *$ & $4.51 * *$ & DF,PB, PPP, GSW \\
\hline
\end{tabular}

Where,

DF-Days to 50per centfloweing; PH-Plant height; PB-Primary branches per plant; PPP- pods per plant, PL-Pod length; SPP- seeds per pod; GSW-Green seed weight; GPY-Green pod yield per plant; SP-Shelling percentage; PRT-Protein content. 
The number of heterotic crosses varied from character to character and the direction and magnitude of heterosis also varied from cross to cross for all the characters. Considerably high heterosis in certain hybrids and low in others revealed that nature of gene action varied with the genetic architecture of the parents. The range of heterobeltiosis and standard heterosis for different characters revealed that high heterosis was observed for primary, pods per plant, seeds per plant, green pod yield per plant. Medium level of heterosis was observed for days to $50 \%$ flowering, plant height, pod length, green seed weight and protein content. While low amount of heterosis were recorded for shelling ratio. The high magnitude of standard heterosis for branches per plant, pods per plant was also reported earlier byPatilet al.,(2005), Pal et al., (2007), Patil and Gosavi, (2007), Sharma, et al.,(2010), Kajale and Ravindrababu, (2012), Patel et al., (2013) and Petheet al., (2017).

A perusal of present study revealed that crosses NC-15-45 X NC-15-41, NCK-15-10 $X$ NC-15-45, and NC-15-45 X NCK-15-10 are top three hybrids for green pod yield per plant and these crosses also showed significant desirable heterosis for protein content. Thus from the present study it can be conclude that most of the hybrids exhibiting significant heterosis for different traits over better parent / standard check. Parents NC-1545,NCK-15-10 and NC-15-41 provide the basic materials for breeding programme for further improvement in yield and yield contributing traits in cowpea. The crosses NC-15-45 X NC-15-41, NCK-15-10 X NC15-45, and NC-15-45 X NCK-15-10 exhibited significant standard heterosis and heterobeltiosis for green pod yield per plant and other yield contributing traits. Thus these crosses may offer for the selection of transgrassive segregant for vegetable purpose cowpea in subsequent generation. Owing to self-pollinated nature of cowpea, heterosis breeding may not be rewarding like in the case of a cross poliinated crops.

\section{Acknowledgement}

The work on cowpea in this paper has been supported financially by DST- INSPIRE, Dept. of Science and Technology, Ministry of Science and Technology, Govt. of India.

\section{References}

Arunachalam, V. 1976. Evaluation of diallel crosses by graphical and combining ability methods. Indian J. Genet., 36: 358-366.

Fonseca, S. and Patterson, F.L. 1968. Hybrid vigour in seven parental dialled crosses in common wheat (Triticum aestivum L.). Crop Sci.2: 85-88.

Jinks, J. L. and Hayman, B. I. 1953. The analysis of diallel crosses. Maize genetics Crops Newsletter, 27: 48-54.

Kajale, D. B. and Ravindarbabu. 2012. Heterosis studies in cowpea [Vigna unguiculata (L.) Walp]. Guj. Agric. Uni. Res. J., 37(1): 7-9

Pal, A. K.; Kumar, S. and Maurya, A. N. 2007. Genet ic study for earliness in cowpea [Vigna unguiculata (L.) Walp].Indian J. Hortic., 64(1): 63-66.

Patel, N.; R. T.; Patel N.; and Koldiya, P. B. 2013.Combining ability study for seed yield in cowpea (Vigna unguiculata (L.)Walp).The Bioscan,8(1): 139-142.

Patil, H. E.; Navale, P. A. and Reddy, N. B. R. 2005.Heterosis and combining ability analysis in cowpea [Vigna unguiculata $(\mathrm{L}$.)Walp].J. Mahaashtra Agric. Uni., 30(1): 88-90.

Patil, H. E. and Gosavi U. S. 2007.Heterosis for yield and yield contributing characters in Cowpea. [Vigna unguiculata (L) Walp.]. International J. Agri. Sci.3(2):326-328.

Pethe, U. B.; Dodiya, N. S.; Bhave, S. G.; 
Dadheech A. and Meghawal, D. R. 2017.Heterosis for yield and yield related traits in cowpea. (Vigna unguiculata L. Walp).J. Pharmacognosy and Phytochemistry, 6(6): 1247-1249.

Sharma, D.; Mehta, N.;Trivedi, J. and Gupata, C. R. 2010.Heterosis Combining Ability And Genetic Divergence In Cowpea [Vigna unguiculata $(\mathrm{L}$. Walp].Vegetable Sci., 37(2): 156-159.

Simmonds, N. W. 1962. Variability in crop plants, its use and conservation.
Biology Review, 37: 314-318.

Steele, W. M. 1976. Cowpeas, [Vigna unguiculata (L.)Walp]. In: Evolution of crop plants, [Eds. R. J. Summerfield and A. H. Bunting], HMSO, London, p. 183-185.

Vavilov, N. I. 1951. The origin, variation, immunity and plant breeding of cultivated plants, Ronald press Corporation, New York, p. 256-257.

\section{How to cite this article:}

Jyoti Kumari and Chauhan, D.A. 2018. Estimation of Heterosis for Green Pod Yield and Attributing Characters in Cowpea (Vigna unguiculata (L.) Walp.). Int.J.Curr.Microbiol.App.Sci. 7(07): 3400-3413. doi: https://doi.org/10.20546/ijcmas.2018.707.395 